\title{
Dynamic critical behaviors in two-dimensional Josephson junction arrays with positional disorder
}

\author{
Jaegon Um, ${ }^{1}$ Beom Jun Kim, ${ }^{2, \text { * }}$ Petter Minnhagen, ${ }^{3}$ M.Y. Choi, ${ }^{4,5}$ and Sung-Ik Lee ${ }^{1,6}$ \\ ${ }^{1}$ National Creative Research Initiative Center for Superconductivity, POSTECH, Pohang 790-784, Korea \\ ${ }^{2}$ Department of Physics, BK21 Physics Research Division, \\ and Institute of Basic Science, Sungkyunkwan University, Suwon 440-746, Korea \\ ${ }^{3}$ Department of Physics, Umeå University, 90187 Umeå, Sweden \\ ${ }^{4}$ Department of Physics and Center for Theoretical Physics, \\ Seoul National University, Seoul 151-747, Korea \\ ${ }^{5}$ Korea Institute for Advanced Study, Seoul 132-722, Korea \\ ${ }^{6}$ Quantum Material Laboratory, Korea Basic Science Institute, Daejon 305-333, Korea
}

(Dated: September 24, 2018)

\begin{abstract}
We numerically investigate dynamic critical behaviors of two-dimensional (2D) Josephson-junction arrays with positional disorder in the scheme of the resistively shunted junction dynamics. Largescale computation of the current voltage characteristics reveals an evidence supporting that a phase transition occurs at a nonzero critical temperature in the strong disorder regime, as well as in the weak disorder regime. The phase transition at weak disorder appears to belong to the BerezinskiiKosterlitz-Thouless (BKT) type. In contrast, evidence for a non-BKT transition is found in the strong disorder regime. These results are consistent with the recent experiment on positionally disordered Josephson-junction arrays; in particular, the critical temperature of the non-BKT transition (ranging from 0.265 down to the minimum 0.22 in units of $E_{J} / k_{B}$ with the Josephson coupling strength $E_{J}$ ), the correlation length critical exponent $\nu=1.2$, and the dynamic critical exponent $z=2.0$ in the strong disorder regime agree with the existing studies of the 2D gauge-glass model.
\end{abstract}

PACS numbers: 74.78.-w, 74.25.Fy, 74.81.Fa

\section{INTRODUCTION}

Phase transitions for the two-dimensional (2D) Josephson-junction arrays (JJAs) with weak positional disorder (i.e., on a slightly disordered lattice) and in a perpendicular magnetic field have attracted much attention. Here positional disorder in the presence of an external magnetic field effectively induces random phase shifts of the magnetic bond angles 1.2 thus the corresponding 2D random gauge $X Y(\operatorname{RG} X Y)$ model, in which the magnetic bond angles are quenched random variables distributed in a certain width, provides a theoretical realization of such a positionally disordered Josephson-junction array (PDJJA) in a magnetic field: Strong magnetic field in the PDJJA corresponds to strong disorder in the RGXY model. It has been observed that sufficiently weak disorder in the RGXY model does not destroy the quasilong-range order present at low temperatures and accordingly, the system undergoes a Berezinskii-KosterlitzThouless (BKT) type transition at a finite critical temperature $T_{c}$, which decreases as the disorder strength is raised up to a critical value. ${ }^{2.3 .4}$ On the other hand, in spite of a number of studies, the strong disorder regime of the RGXY model (and its fully disordered limit corresponding to the gauge-glass model) has resisted adequate understanding. In parallel with the Mermin-Wagner theorem $^{5}$ for the absence of the long-range order in the $X Y$ model, Nishimori ${ }^{\underline{6}}$ has proven the absence of the longrange glass order in the gauge-glass model. However, it should be noted that the vanishing local glass order parameter in the gauge glass is not completely incom- patible with the existence of the superconducting phase at nonzero temperatures. For instance, quasi-long-range glass order ${ }^{7}$ and possibility of a continuous phase transition of an anomalous dimension ${ }^{4}$ have been suggested.

On the one hand, there exist numerical evidences supporting the zero-temperature phase transition: Domainwall renormalization-group studies ${ }^{2.3}$ predict that the RGXY model in the strong disorder regime as well as the gauge-glass model undergoes a phase transition only at zero temperature. For the gauge-glass model, the zerotemperature transition was supported by computations of various quantities such as the current-voltage $(I V)$ characteristics,$\stackrel{8.9}{=}$ the root-mean-square current $\stackrel{10,11}{1}$ the correlation length, ${ }^{12.13}$ the glass susceptibility $\stackrel{10.12 .13}{\rightleftharpoons}$ the autocorrelation function, 11.13 and the phase slip resistance $\stackrel{11}{11}$ A recent numerical consideration of the lowenergy excitations also estimated $T_{c}=0$ in the gaugeglass model $\underline{14}$

In contrast, the finite-size scaling analysis applied to the helicity modulus and the root-mean-square current in the RGXY model with strong disorder demonstrated that the superconducting phase transition of a non-BKT type occurs at finite $T_{c}$, independent of the disorder strength $\stackrel{4}{*}$ In fact evidences for such a finite-temperature transition in the gauge-glass model have been presented in a few numerical studies computing the $I V$ characteristics, $\stackrel{15,16}{,}$ the correlation function,,$\frac{7}{3}$ the glass susceptibility,$\underset{7}{7}$ and the linear resistance ${ }^{17}$ Here it should be noted that critical exponents and $T_{c}$ obtained from the resistively shunted junction (RSJ) simulations in large scales $\frac{16}{}$ agree with other numerical studies of the gauge 
glass,,$\frac{7.17}{r}$ and lead to $T_{c}=0.22$ (in units of $E_{J} / k_{B}$, where $E_{J}$ is the coupling energy and $k_{B}$ the Boltzmann constant), the correlation length critical exponent $\nu=1.2$, and the dynamic critical exponent $z=2.0$. Furthermore, the barrier energy and the associated vortex mobility in the gauge glass $\frac{18}{18}$ implies that superconducting order persists at low but finite temperatures.

Very recently, an experiment has been performed on the PDJJA, 19 and the critical temperature has been measured as a function of the disorder strength, which reveals a finite-temperature transition at strong disorder. Further, in the experiment ${ }^{19}$ the scaling behavior of the $I V$ characteristics is observed consistent with the corresponding numerical results for the gauge glass $\stackrel{15.16}{=}$ indicating the presence of a non-BKT type transition at a finite temperature in the strong disorder regime. A truly remarkable feature of this PDJJA experiment is that a very clear signal of a finite-temperature transition has been obtained for a single disorder realization of a $200 \times 800$ square array. In contrast, typical attempts to settle the issue of the existence of a finite-temperature transition by direct simulations usually presume that a well converged disorder average is necessary. This in turn limits the simulations to small system sizes, so that any conclusion crucially depends on a priori assumptions for the finite-size scaling. The PDJJA experiment in Ref. 19 suggests that one could avoid such a priori assumptions by instead obtaining well converged data for large samples at the price of focussing on a single disorder realization.

In the present paper we test this alternative strategy by performing RSJ simulations and computing the $I V$ characteristics of a large square array of Josephson junctions with one randomly chosen positional disorder realization. In parallel with the experimental results in Ref. 19, we find strong numerical evidence for a finitetemperature transition. In view of the on-going controversy as to the existence of such a phase transition, we discuss the possible implications of our results.

The present paper is organized as follows: In Sec. III we briefly describe the numerical method used in this work, and discuss the relation between the PDJJA and the RGXY model. Section III presents mostly the results for the PDJJA at weak disorder, which is followed by the results at strong disorder in Sec. IV In Sec. V we summarize the results of the present paper in relation with the existing studies.

\section{MODEL AND SIMULATIONS}

With the assumption that the coupling energy is not affected by positional disorder, the Hamiltonian of the PDJJA in the presence of a transverse magnetic field reads $^{25}$

$$
H=-E_{J} \sum_{\langle i j\rangle} \cos \left(\phi_{i j}-A_{i j}\right)
$$

where $\phi_{i j}$ is the phase difference between the superconducting islands at sites $i$ and $j$. When an external magnetic field $\mathbf{B}=B \hat{\mathbf{z}}$ is applied, the magnetic bond angle $A_{i j}$ obtains the form

$$
A_{i j}=\frac{B a^{2} \pi}{\Phi_{0}}\left(x_{j}+x_{i}\right)\left(y_{j}-y_{i}\right),
$$

where $a$ is the lattice constant and $\Phi_{0}$ is the magnetic flux quantum. Due to the positional disorder, the position of the $i$ th island is given by

$$
\mathbf{r}_{i} \equiv\left(x_{i}, y_{i}\right)=\left(x_{i}^{0}+\delta x_{i}, y_{i}^{0}+\delta y_{i}\right),
$$

where $\mathbf{r}_{i}^{0} \equiv\left(x_{i}^{0}, y_{i}^{0}\right)$ represents the ideal position without disorder and $\delta x_{i}$ and $\delta y_{i}$ are random quenched variables uniformly distributed in the interval $[-\Delta, \Delta]$. Henceforth, $\mathbf{r}_{i}, x_{i}, y_{i}, \delta x_{i}$, and $\delta y_{i}$ are all taken to be dimensionless, measured in units of the lattice spacing $a$.

The magnetic frustration $f$ is usually defined as the number of flux quanta per plaquette. There has been intensive research interest in the role of the magnetic frustration in both classical ${ }^{20}$ and quantum ${ }^{21}$ systems. In the absence of positional disorder, every plaquette has an equal area, thus $f$ is constant over the whole system. It is well known that the Hamiltonian (10) in this case is invariant under the transformation $f \rightarrow f \pm 1 \stackrel{20}{2}$ In the present work, however, the plaquette area changes from place to place, and the Hamiltonian loses the above symmetry, invalidating the equivalence between the cases $f$ and $f \pm 1$. We in this work define $f$ as the disorder average (denoted to be $[\cdots]$ ) of the flux through one plaquette

$$
\left[\sum_{p} A_{i j}\right]=2 \pi \frac{B a^{2}}{\Phi_{0}} \equiv 2 \pi f .
$$

The magnetic bond angle $A_{i j}$ in Eq. (2) is correlated with the nearest neighboring bond angles since a change of position $\mathbf{r}_{i}$ gives rise to changes of four magnetic bond angles $A_{i j}$ with site $j$ being neighbors of site $i$. Without such short-range correlations, $A_{i j}$ becomes a random quenched variable characterized by the disorder average $\left[A_{i j}\right]=2 \pi f\left(x_{j}^{0}+x_{i}^{0}\right)\left(y_{j}^{0}-y_{i}^{0}\right)$. In case that $f$ is an integer, one can gauge away the magnetic bond angle, establishing the equivalence with the RGXY model where the average frustration across the whole system vanishes. The variance of the sum of the magnetic bond angles around one plaquette in a PDJJA is given by

$$
\left[\left(\sum_{p} A_{i j}\right)^{2}\right]-\left[\sum_{p} A_{i j}\right]^{2}=\pi^{2} f^{2}\left(\frac{4}{3} \Delta^{2}+\frac{8}{9} \Delta^{4}\right)
$$

whereas the corresponding quantity in the RGXY model reads

$$
\left[\left(\sum_{p} A_{i j}\right)^{2}\right]-\left[\sum_{p} A_{i j}\right]^{2}=\frac{4 \pi^{2}}{3} r^{2}
$$


where $A_{i j}$ has been taken to be uniformly distributed in $[-r \pi, r \pi]$ with the disorder strength $r$. It is to be noted that unless $\Delta$ is close to unity, one obtains

$$
r \approx f \Delta .
$$

Consequently, the disorder strength of the PDJJA in comparison with the RGXY model is measured by $f \Delta$, as found in previous studies of the PDJJA.22.23 It should be noted that the equivalence between the PDJJA and the RGXY model becomes valid only when the external magnetic field yields an integer value of the average magnetic frustration. The relation in Eq. (7) is practically very useful since it provides a convenient way to perform experiments on the 2D RGXY model, in which the disorder strength can be tuned for one sample only by increasing the integral value of $f$.

We below sketch briefly the numerical method adopted in this work. Introducing the twist variable $\mathbf{D} \equiv$ $\left(D_{x}, D_{y}\right)$ for the fluctuating twist boundary conditions $(\mathrm{FTBC})^{24}$ in $L \times L$ arrays, we write $\phi_{i j}$ in Eq. (1) as

$$
\phi_{i j}=\theta_{i}-\theta_{j}-\mathbf{r}_{i j}^{0} \cdot \mathbf{D},
$$

where $\mathbf{r}_{i j}^{0} \equiv \mathbf{r}_{j}^{0}-\mathbf{r}_{i}^{0}$ and $\theta_{i}$ is the phase angle. Since the effects of positional disorder are introduced only through magnetic bond angles, we use the RSJ dynamics combined with the FTBC as follows: Using the local current conservation, the equation for the phase angle at site $i$ is given by

$$
\dot{\theta}_{i}=-\sum_{j} G_{i j} \sum_{k}^{\prime}\left[\sin \left(\phi_{j k}-A_{j k}\right)+\eta_{j k}\right],
$$

where time has been measured in units of $\hbar / 2 e i_{c} R$ with the single-junction critical current $i_{c}=2 e E_{J} / \hbar$ and the shunt resistance $R$ (see Ref. 24 for details), $G_{i j}$ is the square-lattice Green function, $\sum_{k}{ }^{\prime}$ denotes the summation over the four nearest neighbor sites of $j$, and $\eta_{j k}$ is the dimensionless thermal noise current satisfying $\left\langle\eta_{i j}\right\rangle=0$ and

$$
\left\langle\eta_{i j}(t) \eta_{k l}(0)\right\rangle=2 T\left(\delta_{i k} \delta_{j l}-\delta_{i l} \delta_{j k}\right) \delta(t)
$$

with the temperature $T$ in units of $E_{J} / k_{B}$. In order to use the efficient fast Fourier transform, we modify the periodic boundary condition for $\theta_{i}$ according to

$$
\begin{aligned}
\theta_{i+L \hat{\mathbf{x}}} & =\theta_{i}+2 \pi f L \delta y_{i} \\
\theta_{i+L \hat{\mathbf{y}}} & =\theta_{i},
\end{aligned}
$$

where $2 \pi f L \delta y_{i}$ is fixed in time and thus $\dot{\theta}_{i+N \hat{\mathbf{x}}}=\dot{\theta}_{i}$. Here the usual periodic boundary conditions $\left(\theta_{i+L \hat{\mathbf{x}}}=\right.$ $\left.\theta_{j+L \hat{\mathbf{y}}}=\theta_{i}\right)$ are not applicable since $A_{i j} \neq A_{i+L \hat{\mathbf{x}}} j+L \hat{\mathbf{x}}$ for the PDJJA.

We consider the system under uniform external currents injected along the $x$-direction. The Josephson relation $2 e V_{x} / \hbar=\dot{\phi}_{i+N \hat{\mathbf{x}} i}$, where $V_{x}$ is the voltage drop across the sample, together with the global current conservation condition, 24 leads to the equations of motion for the twist variables $D_{x}$ and $D_{y}$ in the form

$$
\begin{aligned}
& \dot{D}_{x}=\frac{1}{L^{2}} \sum_{\langle i j\rangle_{x}} \sin \left(\phi_{i j}-A_{i j}\right)+\eta_{D_{x}}-i_{d}, \\
& \dot{D}_{y}=\frac{1}{L^{2}} \sum_{\langle i j\rangle_{y}} \sin \left(\phi_{i j}-A_{i j}\right)+\eta_{D_{y}},
\end{aligned}
$$

where $\sum_{\langle i j\rangle_{x(y)}}$ denotes the directed sum over nearest neighboring bonds in the $x(y)$ direction, $i_{d}$ is the external (driving) current density in units of the single-junction critical current $i_{c}$, and $\eta_{D_{x}}\left(\eta_{D_{y}}\right)$ is the thermal noise current for $D_{x}\left(D_{y}\right)$ satisfying $\left\langle\eta_{D_{x}}\right\rangle=\left\langle\eta_{D_{y}}\right\rangle=0$ and

$$
\left\langle\eta_{D_{x}}(t) \eta_{D_{x}}(0)\right\rangle=\left\langle\eta_{D_{y}}(t) \eta_{D_{y}}(0)\right\rangle=2\left(T / L^{2}\right) \delta(t) .
$$

We numerically integrate the equations of motion given by Eqs. (9) and (12), and compute the $I V$ characteristics with the average (dc) voltage $V \equiv L v \equiv-L\left\langle\dot{D}_{x}\right\rangle$, where $v$ denotes the voltage drop per junction in units of $i_{c} R$.

\section{RESULTS FOR WEAK DISORDER}

Our simulation scheme requires sufficiently precise data for a large sample and a large disorder strength. We find that RSJ simulations of the PDJJA on a $128 \times 128$ square lattice can be made to meet all these three requirements simultaneously, and thus use this lattice size in the present investigation.

To probe phase transitions in weak and strong disorder regimes, we compute the $I V$ characteristics at various temperatures and values of $f$ with the latter controlling the disorder strength (see Sec. III). In our simulations we set the parameter for positional disorder equal to $\Delta=$ 0.2 . Since $r \approx f \Delta$ and the critical disorder strength in the RGXY model is believed to be $r_{c} \approx 0.4,2.3 .4$ we vary the average frustration $f$ from 1 to 4 to cover both weakly ( $f=1$ with $f \Delta \approx 0.2<r_{c}$ ) and strongly $(f \geq 2$ with $f \Delta>r_{c}$ ) disordered cases.

Figure 1 presents the dc resistance $v / i_{d}$ (in units of the shunt resistance $R$ ) versus the temperature $T$ at various frustrations $f=1,2,3$, and 4 . When $f=1$, corresponding to the weakly disordered case, the resistancetemperature (RT) curves in Fig. 1] disclose that the system undergoes a phase transition around $T=0.6$, which is lower than $T_{c}=0.89$ in a regular JJA. ${ }^{24}$ For strong disorder $(f \geq 2)$, phase transitions are observed to occur near $T=0.2$, hardly depending on the frustration.

In order to determine the transition temperature in a more accurate way and to understand the dynamic critical behavior in detail, we next investigate the $I V$ characteristics at various values of $f$ with the help of the scaling form suggested in Ref. 26. In two dimensions, the current density and the electric field behave as $J \sim T / \xi$ and $E \sim \xi^{-1-z}$, respectively, with the correlation length $\xi$. 


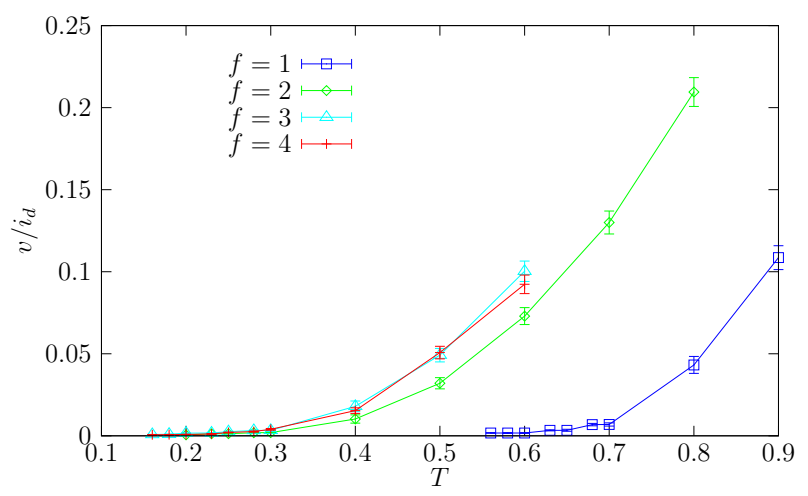

FIG. 1: (Color online) Resistance $v / i_{d}$ (in units of $R$ ) versus temperature $T$ (in units of $E_{J} / k_{B}$ ) for various frustrations in positionally disordered Josephson-junction arrays. As the frustration is raised from $f=1$ to $f=2$, the effective disorder strength becomes larger, reducing the critical temperature. Larger values of $f(=3,4)$ yield only insignificant variations of the critical temperature.

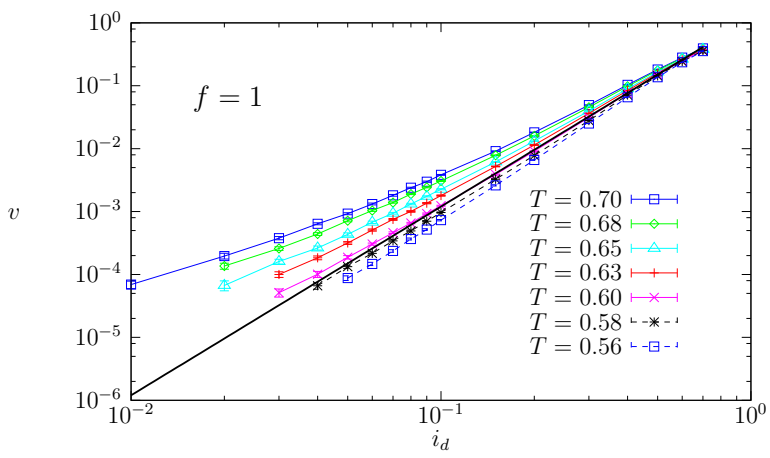

FIG. 2: (Color online) $I V$ curves $\left(v\right.$ versus $i_{d}$ ) for $f=1$ in $\log$ scales at various temperatures $T$ (in units of $E_{J} / k_{B}$ ). At $T \lesssim 0.6$, curves fit well to the power-law form $v \approx i_{d}^{z+1}$, with the solid line representing the case $z=2$. At higher temperatures, $I V$ curves bend upwards, indicating a BKTtype transition.

In the case $T_{c} \neq 0$, one replaces $T$ in $J \sim T / \xi$ by $T_{c}$ near criticality, and obtains accordingly $J \sim \xi^{-1}$. In this work, $i_{d}$ and $v$ correspond to the current density and the electric field, respectively, and yield the scaling form

$$
v=i_{d} \xi^{-z} F_{ \pm}\left(i_{d} \xi\right)
$$

with the scaling function $F_{ \pm}$above/below $T_{c}$. At $T=T_{c}$, the correlation length $\xi$ diverges and Eq. (14) leads to $v \approx i_{d}^{z+1}$.

In Fig. 2] we display the $I V$ characteristics for $f=1$, corresponding to the weak disorder regime, near $T=$ $T_{c} \approx 0.6$ (see the RT curve for $f=1$ in Fig. (1). Due to the finite-size effects, $I V$ curves exhibit the Ohmic behavior in the small current region as $i_{d} \rightarrow 0 \stackrel{24}{\underline{24}}$ The curves at $T \lesssim 0.6$ fit well to the power-law form $v \sim i_{d}^{a}$ : At $T \approx 0.6$, we have $a \approx 3$, and the lower $T$, the larger $a$. In contrast, the $I V$ curves at higher temperatures $(T \gtrsim 0.6)$ clearly exhibit upward curvature, indicating the existence

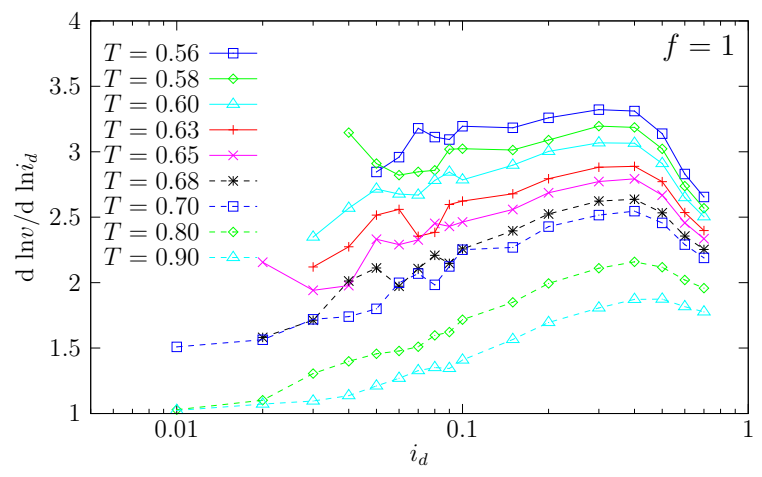

FIG. 3: (Color online) Slope $d \ln v / d \ln i_{d}$ of the $I V$ curve versus the driving current $i_{d}$ at various temperatures (in units of $\left.E_{J} / k_{B}\right)$. Due to finite-size effects, the slope first increases with $i_{d}$, then decreases toward the Ohmic value, unity, thus forming a peak. In a broad range of temperatures below $T_{c} \approx$ 0.7 , the peak position $i_{d}^{*}$ does not change substantially while it shifts to higher currents beyond $T_{c}$. Such behavior of the peak position is consistent with the BKT-type transition.

of a finite current scale (and thus of a finite length scale). From the relation $a=z+1$ between the dynamic critical exponent $z$ and the nonlinear $I V$ exponent $a$, we thus reach the conclusion that a phase transition of the BKT nature occurs at $T_{c} \approx 0.6$ with $z \approx 2$. It should be noted that the scaling form in Eq. (14) is valid when the correlation length diverges only at $T_{c}$ and becomes smaller as the temperature is varied (either lowered or raised) from $T_{c}$. Accordingly, when the phase transition is of the BKT type, characterized by the diverging length scale in the whole low-temperature phase, only high-temperature $I V$ curves collapse to the scaling form, while the lowtemperature part cannot be made to collapse due to the lack of the length scale $\xi$.

Alternatively, one can also plot the slopes of $I V$ curves, given by $d \ln v / d \ln i_{d}$, versus the driving current $i_{d}$, as shown in Fig. 3 to confirm the BKT nature of the transition 27 As $i_{d}$ is reduced, all curves should eventually crossover to the Ohmic behavior characterized by the unit slope $\left(d \ln v / d \ln i_{d}=1\right)$ due to the finite-size effects. The peak position $i_{d}^{*}$ of each curve in Fig. 3] measures a characteristic current scale, which is inversely proportional to the length scale in the system, i.e., $i_{d}^{*} \approx i / \xi$. The correlation length $\xi$ increases as $T$ approaches $T_{c}$ from high temperatures. Near $T_{c}$ in the high-temperature phase and also in the whole low-temperature phase, the correlation length becomes larger than the size of the system. In this case, the relevant length scale of the system is not the correlation length but the linear size $L$ of the system, leading to $i_{d}^{*} \sim 1 / L$ independent of the temperature. In Fig. 3 the peak position $i_{d}^{*}$ has almost the same value around 0.4 as $T$ is increased from below up to $T \approx 0.7$; then $i_{d}^{*}$ appears to drift away toward larger values as $T$ is increased further beyond $T=0.7$, which is in good agreement with what we expect for the BKT-type phase transition. We emphasize again that for a finite 
system $i_{d}^{*}$ starts to move not at the true critical temperature but at a temperature higher than $T_{c}$ when the correlation length becomes comparable to $L$. Between $T=0.63$ and $0.6, d \ln v / d \ln i_{d} \approx 3$ at $i_{d}^{*}$, which, combined with the power-law decay form in Fig. 2 makes us conclude that the BKT transition occurs at $T_{c} \approx 0.6$ (in accord with the RT curve in Fig. 1) with the dynamic critical exponent $z \approx 2$. This analysis of the $I V$ curves clearly reveals that the phase transition of the BKT type occurs in the weak positional disorder regime of the PDJJA, consistent with experiment ${ }^{19}$ and previous numerical studies of RGXY model 2.3 .4

\section{RESULTS FOR STRONG DISORDER}

In the strong disorder regime $(f \geq 2)$, we show in Fig. [4 the $I V$ curves for $f=$ (a) 2, (b) 3 , and (c) 4 . It is clearly observed that for each value of $f$, there exists a welldefined temperature $T_{c}$ at which the $I V$ curve shows a power-law behavior, manifesting the absence of a length scale at criticality. The critical temperature estimated from Fig. [4 is $T_{c}=$ (a) 0.25, (b) 0.23 , and (c) 0.23 for $f=2,3$, and 4 , respectively, in agreement with Fig. [1 It is also shown in Fig. 4 that the $I V$ curves at $T_{c}$ fit well to the form $i_{d}^{z+1}$ with the dynamic critical exponent $z=2$. The $I V$ curves in Figs. 4 (a), (b), and (c) all bend upward above $T_{c}$, indicating that the PDJJA is in the high-temperature normal phase, while the opposite downward curvature below $T_{c}$ implies the superconducting phase in the limit of $i_{d} \rightarrow 0$.

To find nature of the phase transition and the critical temperature together with critical exponents in the strong disorder regime of the PDJJA, we employ the scaling form in Eq. (14), which, with the correlation length $\xi \sim\left|T-T_{c}\right|^{-\nu}$, reads

$$
\frac{v}{i_{d}\left|T-T_{c}\right|^{z \nu}}=F_{ \pm}\left(i_{d}\left|T-T_{c}\right|^{-\nu}\right) .
$$

Figures 4 (d)-(f) show that data points in the $I V$ characteristics [in Figs. 目 (a)-(c)] collapse into two function $F_{ \pm}$in Eq. (15); this confirms that for given value of $f$, there exists a finite-temperature phase transition of a non-BKT type. The critical temperature changes from $T_{c} \approx 0.265$ for $f=2$ to $T_{c} \approx 0.22$ for $f=3$ and 4 , which is consistent with the experiment on the PDJJA ${ }^{19}$ and numerical studies of the RGXY mode ${ }^{\underline{4}}$ and the gaugeglass model ${ }^{7.15,16.17 .18}$ On the other hand, in all three

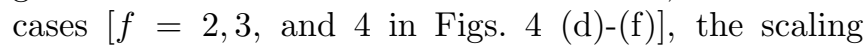
collapse is achieved with $\nu=1.2$ and $z=2.0$, implying that the nature of the transition in the strong disorder regime remains unchanged as the disorder strength is increased. Furthermore, the dynamic critical exponent $z=2.0$ agrees with the studies of dynamic behavior in the gauge-glass mode ${ }^{7.16 .17}$ as well as with the experimental results 19 . The critical exponent $\nu=1.2$ obtained in this work is again consistent with the previous numerical results 4.7 .16 .17 but not with the experimental result for the PDJJA, ${ }^{19}$ the origin of which is not clear at this stage.

We next investigate slopes of the $I V$ curves for $f=4$ in the same manner as in Sec. III for the weak disorder case for $f=1$. In Fig. [5 the slope $d \ln v / d \ln i_{d}$ is plotted as a function of $i_{d}$ at various temperatures around $T_{c}$. Note that finite-size effects yield the Ohmic behavior, $d \ln v / d \ln i_{d} \approx 1$, as $i_{d} \rightarrow 0$. It is observed that the curves at $T=0.23$ and 0.20 are almost horizontal in broad ranges of the external currents, indicating scalefree behavior and in turn the existence of a phase transition with the dynamic critical exponent $z \approx 2.0$, consistent with the finding in Fig. 目(f) for $f=4$. Furthermore, the fact that the peak position changes rather abruptly from large currents to smaller currents at $T=0.23$ to 0.20 implies that the phase transition is of a non-BKT type, namely, the $I V$ data are well scaled to two different functions, $F_{ \pm}$in Eq. (15) above/below $T_{c}$.

In view of the ongoing controversy about the existence of a finite-temperature transition of a non-BKT character for strong disorder, one might ask what our results really imply. First of all, our evidence has been obtained for a finite sample with one disorder realization (albeit a very large one in comparison with those in most of earlier investigations). Does the phase transition survive in the large system size limit? Since a single positional disorder for a finite system can be periodically repeated, we can obtain an arbitrarily large system by just adding new squares with the same single disorder realization. Such an infinite system constructed from a single disorder realization will, to our belief, most certainly have a finite-temperature transition of a non-BKT type. This means that the phase transition does exist per se. Suppose that we instead choose an arbitrarily large system and generate the disorder in the same random way as we have done for our $128 \times 128$ sample. Would the phase transition still survive? Here we find that various randomly generated disorder realizations for the $128 \times 128$ system yield numerically very similar results, indicating that for the size $L=128$ there is already a large amount of disorder self-averaging. This again suggests that our results will survive in the large $L$ limit, i.e., for the PDJJA with uniformly distributed disorder, chosen randomly, of strength $\Delta=0.2$. In order to check the finite-size effect on the self-averaging property in a more careful way, we have also computed voltages at $T=0.16$ in the strong disorder regime $(f=4)$ for smaller sizes ( $L=16,32,64$, and 128) at two different disorder realizations : The difference between voltages obtained from different disorder realizations is found to decrease as $L$ is increased, and becomes negligibly small at $L=128$. This indicates that indeed the self-averaging effect becomes clear beyond $L=128$. The next question is then whether our results also carry over to the RGXY model and the gauge-glass model. Here the evidence is more circumstantial and based, on the one hand, on the strong connection between the PDJJA and the RGXY and random gauge-glass models ${ }^{1.2}$ and, on the other hand, on the 

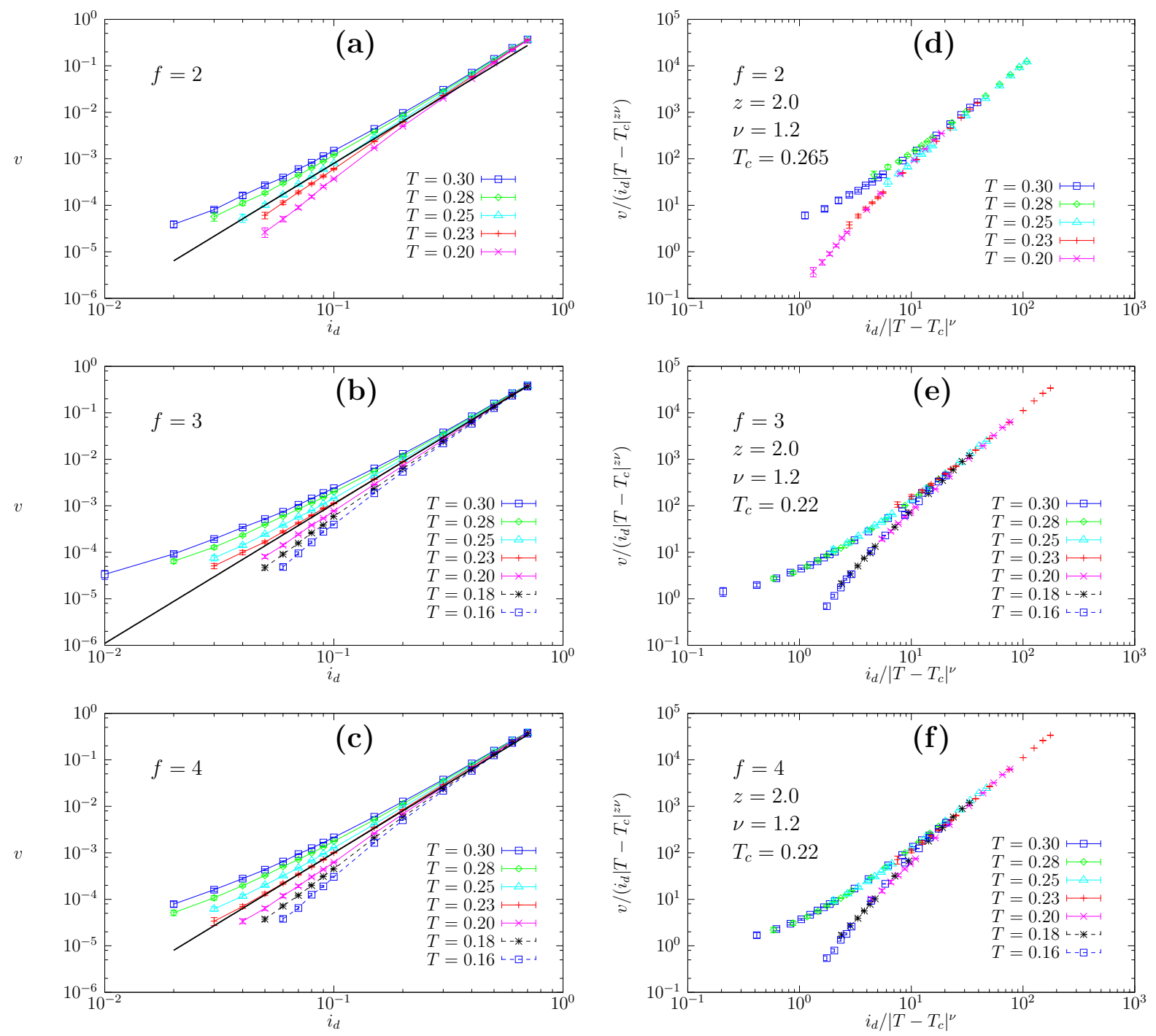

FIG. 4: (Color online) $I V$ characteristics $\left(v\right.$ versus $i_{d}$ ) at various temperatures for $f=(\mathrm{a}) 2$, (b) 3 , and (c) 4 , and the corresponding scaling plots in (d)-(f). The solid lines in (a), (b), and (c) represent the power-law decay form $v \approx i_{d}^{z+1}$ with $z=2$. A well-defined temperature $T_{c}$ separates the $I V$ curves into two groups, one bending upwards and the other downwards. All data points in the corresponding $I V$ characteristics are made to collapse into scaling functions, as shown in (d)-(f), with $T_{c}=0.265,0.22$, and 0.22 , respectively. In all the three cases (d)-(f), the same critical exponents $\nu=1.2$ and $z=2.0$ are used, implying that the transitions in the strong disorder regime $(f=2,3$, and 4$)$ belong to the same universality class.

strong similarity of the present results to some earlier results obtained from the latter models $\stackrel{4,7,16,17}{\underline{4}}$

\section{SUMMARY}

The existence of a finite-temperature phase transition in the strong disorder regime of the PDJJA and the 2D RGXY model, including the fully disordered case of the 2D gauge-glass model, are still in an intensive debate. This work has been motivated by the very recent experiment on $800 \times 200$ Josephson junction arrays with positional disorder $\frac{19}{\underline{n}}$ and explored numerically the dynamic critical behavior of the PDJJA in transverse magnetic fields. Adopting the RSJ dynamics $\stackrel{24}{,}$ we have computed the $I V$ characteristics of the PDJJA with the positional disorder parameter $\Delta=0.2$ for frustration $f=1,2,3$ and 4 . The relation $r \approx f \Delta$ between the disorder strength $r$ in the 2D RGXY model and the parameter $\Delta$ in the PDJJA, combined with the critical disorder strength $r_{c} \approx 0.4,2.3 .4$ implies that the PDJJA for $f=1$ corresponds to the weakly disordered case while strong frustration $(f \gtrsim 2)$ puts the PDJJA in the strong disorder regime. The scaling analysis ${ }^{26}$ of $I V$ curves and their slopes 27 has revealed clear evidence for $T_{c} \neq 0$ in the PDJJA with strong disorder, which agrees with the experiment on the PDJJA ${ }^{19}$ as well as previous numerical studies of the $\operatorname{RGXY}$ mode $\mathbb{1}^{4}$ and the gauge-glass 


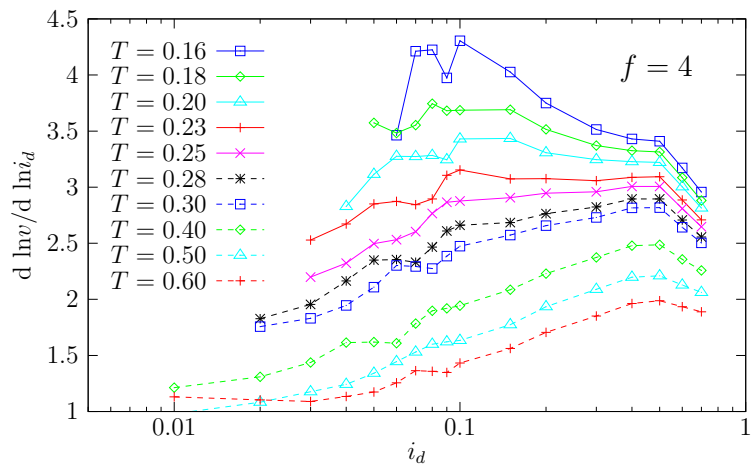

FIG. 5: (Color online) Slope $\left(d \ln v / d \ln i_{d}\right)$ of $I V$ curves against $i_{d}$ for $f=4$, corresponding the strong disorder regime of the PDJJA, at various temperatures $T$ (in units of $E_{J} / k_{B}$ ). Power-law behavior appears at $T=0.23$ to 0.20 with the dynamic critical exponent $z \approx 2$, indicating the existence of a phase transition with a diverging length scale only at $T_{c}$.

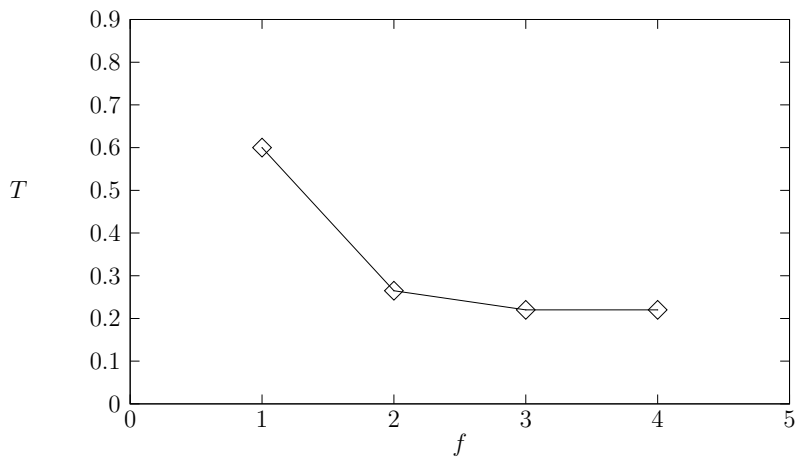

FIG. 6: Phase diagram on the plane of temperature $T$ (in units of $E_{J} / k_{B}$ ) and frustration $f$ (controlling the disorder strength). The solid line, separating the high-temperature normal phase from the low-temperature superconducting phase, is only a guide to the eye. The critical temperature saturates to a finite nonzero value $T_{c} \approx 0.22$ as $f$ is increased. Below $T_{c}$, depending on the disorder strength, there exist two different superconducting phase: a BKT-like phase for $f=1$ and a non-BKT type one for $f \gtrsim 2$. model $\underline{7,15,16,17,18}$

Figure 6] exhibiting the phase diagram on the plane of the temperature and frustration summarizes the results of the present work. The critical temperature $T_{c}$ is observed to reduce rapidly as the average frustration $f$, controlling the disorder strength, is increased in the weak disorder region. It then appears to saturate toward a constant value $T_{c}=0.22$ in the strong disorder regime. Below the phase boundary separating the superconducting phase at low temperatures and the normal phase at high temperature, there exist two different superconducting orders, according to the disorder strength: In the weak disorder regime, e.g., $f=1$, the superconducting state is the low-temperature BKT phase characterized by the divergence of the correlation length. On the other hand, for strong disorder $(f \gtrsim 2)$, the transition is of the nonBKT type with the well-defined correlation length critical exponent $\nu=1.2$, consistent with the value obtained previously for the 2D gauge-glass model7.16.17 but inconsistent with the experimental finding $\nu=2.0 \pm 0.3 .^{19}$ The origin of this discrepancy is not clear at present and needs more detailed investigation in the future study. Finally, we point out that the resemblance between the phase diagram in Fig. [6] and the corresponding diagram obtained in the recent experiment on the PDJJA ${ }^{19}$ is striking, which is also in very good agreement with the numerical study of the $2 \mathrm{D}$ random gauge $X Y$ model $^{4}$

\section{Acknowledgments}

This work was supported by the Korea Research Foundation Grant funded by the Korean Government (MOEHRD) KRF-2005-005-J11903 (B.J.K.), through the National Research Center for Systems Bio-Dynamics (M.Y.C.), and through the Creative Research Initiatives Program (S.-I.L.).
* Corresponding author; beomjun@skku.edu

1 M. G. Forrester, and S. P. Benz, and C. J. Lobb, Phys. Rev. B 41, 8749 (1990); S. E. Korshunov, ibid. 48, 1124 (1993).

2 J. M. Kosterlitz and M. V. Simkin, Phys. Rev. Lett 79, 1098 (1997).

3 N. Akino and J. M. Kosterlitz, Phys. Rev. B 66, 054536 (2002).

4 P. Holme, B. J. Kim, and P. Minnhagen, Phys. Rev. B 67, 104510 (2003).

${ }^{5}$ N. D. Mermin and H. Wagner, Phys. Rev. Lett. 17, 1133 (1966); P. Bruno, ibid. 87, 137203 (2001).

${ }^{6}$ H. Nishimori, Physica A 205, 1 (1994).

7 M. Y. Choi and S. Y. Park, Phys. Rev. B 60, 4070 (1999).

8 R. A. Hyman, M. Wallin, M. P. A. Fisher, S. M. Girvin, and A. P. Young, Phys. Rev. B 51, 15304 (1995).

${ }^{9}$ E. Granato Phys, Rev. B 58, 11161 (1998).

10 H. G. Katzgraber and A. P. Young, Phys. Rev. B 66, 224507 (2002).

11 M. Nikolaou and M. Wallin, Phys. Rev. B 69, 184512 (2004).

12 H. G. Katzgraber, Phys. Rev. B 67, 180402(R) (2003).

13 H. G. Katzgraber and I. A. Campbell, Phys. Rev. B 69, 094413 (2004).

14 L.-H. Tang and P. Tong, Phys. Rev. Lett 94, 207204 (2005).

15 Y.-H. Li, Phys. Rev. Lett 69, 1819 (1992).

16 Q.-H. Chen, A. Tanaka, and X. Hu, Physica B 329, 1413 (2003).

17 B. J. Kim, Phys, Rev. B 62, 644, (2000). 
18 P. Holme and P. Olsson, Europhys. Lett 60, 439 (2002).

19 Y.-J. Yun, I.-C. Baek, and M.-Y. Choi, cond-mat/0509151 Y.-J. Yun, Ph.D. dissertation, Sungkyunkwan University, 2005 (unpublished).

20 S. Teitel and C. Jayaprakash, Phys. Rev. B 27, 598 (1983); W. Y. Shih and D. Stroud, ibid. 28, 6575 (1983); M. Y. Choi and S. Doniach, ibid. 31, 4516 (1985); T. C. Halsey, J. Phys. C 18, 2437 (1985); B. J. Kim and P. Minnhagen, ibid. 60, 588 (1999).

${ }^{21}$ Y. Tan and D. J. Thouless, Phys. Rev. B 46, 2985 (1992); T. K. Kopeć and T. P. Polak, ibid. 66, 094517 (2002); B. J. Kim, G. S. Jeon, M.-S. Choi, and M. Y. Choi, ibid. 58, 14524 (1998).

22 E. Granato and J. M. Kosterlitz, Phys. Rev. B 33, 6533 (1986).
23 M. Y. Choi, J. S. Chung, and D. Stroud, Phys. Rev. B 35 1669 (1987).

24 B. J. Kim, P. Minnhagen, and P. Olsson, Phys. Rev. B 59, 11506 (1999)

25 We assume that a change of the central position of a superconducting island in a Josephson junction array does not alter the Josephson coupling strength, which is related with the tunneling amplitude of Cooper pairs (see Ref. 19 for more details).

26 D. S. Fisher, M. P. A. Fisher, and D. A. Huse, Phys. Rev. B 43, 130 (1991).

27 K. Medvedyeva, B. J. Kim, and P. Minnhagen, Phys. Rev. B 62, 14531 (2000). 